\title{
Indirect (chronic) self-destructiveness and modes of suicide attempts
}

Konstantinos Tsirigotis ${ }^{1}$, Wojciech Gruszczynski², Marta Tsirigotis-Wołoszczak ${ }^{3}$

\begin{abstract}
${ }^{1}$ Department of Psychology, The Jan Kochanowski University of Humanities and Sciences, Piotrków Trybunalski, Poland

${ }^{2}$ Neurotic and Stress Related Disorders Clinic, Medical University of Lodz, Lodz, Poland

${ }^{3}$ Division of Medicinal Chemistry and Microbiology, Chemistry Department, University of Technology, Wroclaw, Poland
\end{abstract}

Submitted: 23 September 2009

Accepted: 17 December 2009

Arch Med Sci 2010; 6, 1: 111-116

DOI 10.5114/aoms.2010.13517

Copyright ( 2010 Termedia \& Banach

\section{Abstract}

Introduction: Suicides and suicide attempts may be considered as manifestations not only of direct self-destructiveness, but also of indirect (chronic) selfdestructiveness. The aim of this work is to assess the relation between intensity of indirect self-destructiveness and suicide attempt modes.

Material and methods: The research was conducted among 147 persons after suicide attempts (114 women and 33 men) aged 14-33 years. The Polish version of the "Chronic Self-Destructiveness Scale" by Kelley (CS-DS) in Suchańska's adaptation was used.

Results: Examined persons applied several modes of committing suicide: pharmacological drugs, exsanguination, hanging, jumping from a height, asphyxia, poisoning, and throwing oneself under a moving car. The most frequent are pharmacological drugs (42.31\%) and exsanguination (25.60\%); the rarest is throwing oneself under a car (1.28\%). Relations between indirect self-destructiveness and the following suicide attempt modes were found: pharmacological drugs $(0.366$, $p=0.00001)$, poisoning $(0.667, p=0.01)$, and throwing oneself under a car $(0.542$, $p=0.04)$. There were found three clusters regarding the intensity of indirect selfdestructiveness (low, medium and high). The largest differences concerned hanging, jumping from a height (medium CS-DS), poisoning and throwing oneself under a car (high CS-DS). There is a relation between indirect self-destructiveness and recurrence of suicide attempts (Kendall Tau $=0.217, p=0.007$ ); the intensity of indirect self-destructiveness differentiates the risk of consecutive suicide attempts $(\mathrm{F}=2.871, p=0.05)$.

Conclusions: The implications of the findings are mainly of preventive and therapeutic nature. In the preventative aspect, the subject of interest should be not only persons with a tendency to the active form of indirect selfdestructiveness, but also persons exhibiting its passive form.

Key words: indirect (chronic) self-destructiveness, modes of suicide attempts.

\section{Introduction}

The phenomenon of self-harming has been well known for a long time. Suicide is now among the five top causes of death in youth worldwide [1]. Suicide is one of the leading causes of death worldwide, mortality from suicide being approximately $2 \%$. Attempted suicide appears to be a major risk factor for suicide completion [2]. For these reasons, suicide attempts have become an important public health issue throughout the world.

To date there have been examined some of the personality traits of persons after suicide attempts, e.g. persons with a history of attempted

\author{
Corresponding author: \\ dr. Konstantinos Tsirigotis \\ Department of Psychology \\ Jan Kochanowski University \\ of Humanities and Sciences \\ Stowackiego 114/118 \\ 97-300 Piotrków Trybunalski, \\ Poland \\ Phone: +48 44 732-74-00, \\ ex. 141 \\ Fax: +48 43 843-91-60 \\ E-mail: \\ psyche1@onet.eu
}


suicide were assessed for anger, aggression, temperament/character, etc. [2]; it was found that among the factors linked with increased risk of suicide attempts are helplessness and hopelessness [3], also alienation and a feeling of not being understood by others [4].

Hitherto suicides and suicide attempts have been treated mainly as categories (types, manifestations) of direct self-destructiveness. But suicides and suicide attempts can also be manifestations of indirect (chronic) self-destructiveness (not only of the direct one); suicide attempts (and suicides) are related to a psychological trait or psychological syndrome such as indirect self-destructiveness [5]. It is worth considering indirect self-destructiveness as one of the psychological mechanisms of committing suicide and suicide attempts.

While direct self-destructive behaviours (suicides, self-mutilation, etc.) are obvious and indubitable, less acute and more "subtle" forms of harming oneself or shortening and/or worsening life quality are not immediately and directly perceptible (risky behaviours, addictions, omissions, carelessness, etc.). Usually less attention is dedicated to them, especially as most of them are considered to be commonly (or at least frequently) occurring behaviours, and are thus called "normal".

Chronic self-destructiveness is defined as behaviour involving the generalised tendency to engage in acts that increase the probability of experiencing future negative consequences and/or reduce the probability of attaining future positive ones [6]. Indirect (chronic) self-destructiveness is also considered as behaviour whose probable negative consequences are mediated by additional factors, and the relation between behaviour and harm is considered as a probable one. Indirect (chronic) self-destructiveness, implied this way, includes not only undertaking and abandoning (commission or omission of) acts; it is also concerns involvement in dangerous and aggravated risk situations, or neglecting one's health and safety $[7,8]$.

Table I. Suicide attempt modes

\begin{tabular}{|cccc|}
\hline Rank & Suicide attempt mode & $n$ & $\%$ \\
\hline 1. & Pharmacological drugs & 99 & 42.31 \\
\hline 2. & Exsanguination & 60 & 25.64 \\
\hline 3. & Hanging & 39 & 16.67 \\
\hline 4. & Jumping from a height & 21 & 8.97 \\
\hline 5. & Asphyxia & 9 & 3.85 \\
\hline 6. & Poisoning & 3 & 1.28 \\
\hline 7. & Throwing oneself under & 3 & 1.28 \\
\hline & a moving car & 234 & 100.00 \\
\hline & Total &
\end{tabular}

The aim of this work is to study the associations between the intensity of indirect (chronic) selfdestructiveness and modes of suicide attempts.

\section{Material and methods}

This study was carried out among persons who attempted suicide by various modes and were admitted to hospital for this reason. The study sample consisted of 147 participants (114 women and 33 men) aged between 14 and 33. A decisive majority of study participants were in the care of treatment centres for mental disorders (in- and outpatients) due to suicide attempts. Examined patients were not diagnosed as having psychotic disorders or mental retardation.

Examination was anonymous, and participation was voluntary. Obtaining permission from the institution management and examined individuals was necessary for participation in the research.

To examine indirect self-destructiveness, the Polish version of the "Chronic Self-Destructiveness Scale” by Kelley (CS-DS) in Suchańska's adaptation was performed.

To assess chronic (indirect) self-destructiveness as a generalised tendency, Kelley designed a research instrument including four groups or categories of behaviours: carelessness, poor health maintenance, evidence of transgression, and lack of planfulness. The ultimate version consists of an internally coherent set of 52 questions equally for women and men [6]. The Polish version of the scale, like the original one, is characterized by high reliability and validity [7, 8], and also includes helplessness and passivity.

The quantitative data obtained were subjected to statistical analysis by means of the statistical package Statistica PL 8.0 for Windows [9]. The data were analysed using correlation coefficient, hierarchical cluster analysis, and ANOVA. Values were expressed as mean $\pm \mathrm{SD} ; p \leq 0.05$ was considered significant.

\section{Results}

Scores gained by suicide attempters in CS-DS contain in the upper limit of the range of average scores, despite professional (medical and psychological) care: mean 154.041, SD 22.919.

Participants applied several modes of committing suicide: pharmacological drug abuse, exsanguination, hanging, jumping (from a height), asphyxia, poisoning and throwing oneself under a moving car (Table I).

The most often chosen modes are pharmacological drug abuse (42.31\%) and exsanguination (25.64\%); the rarest is throwing oneself under a moving car (1.28\%). Predominantly chosen modes of committing suicide are distinctly different from 
those found in other studies, e.g. hanging $[10,11]$ In the study sample, within the framework of this work, use of firearms as suicide attempts was not found.

In order to assess relations between indirect selfdestructiveness and suicide attempt modes, correlation analysis, hierarchical cluster analysis, and ANOVA were conducted.

As a result of the performed correlation analysis (Table II), statistically significant correlations between indirect self-destructiveness and mode of suicide attempt were found for: pharmacological drugs $(0.366, p=0.00001)$, poison $(0.667, p=0.01$ ), and throwing oneself under a moving car (0.542, $p=0.04)$.

As can be seen, indirect self-destructiveness shows significant correlations with the most frequently (pharmacological drugs) and infrequently (poison and car) chosen suicide attempt modes.

In order to verify whether indirect selfdestructiveness intensity may differentiate suicide attempters with respect to choice of suicide mode, hierarchical cluster analysis was performed, and as a result, three clusters emerged: cluster 1 (high intensity of indirect self-destructiveness), cluster 2 (medium intensity) and cluster 3 (low intensity; Table III).

As can be seen in Table III, the most numerous is cluster 3 (low indirect self-destructiveness intensity), and the least numerous is cluster 1 (high indirect self-destructiveness intensity).

In Table IV the distribution of particular suicide attempt modes is presented within each cluster. As shown in Table IV, the rank order of suicide attempt modes (regarding the frequency of selection within each cluster) was the same as it was in the whole sample only in the case of cluster 1 (high scores in CS-DS). In other words, participants from cluster 1 "take the lead", forming the pattern and structure of suicide attempt modes in the whole study population. In the remaining clusters, the rank order has changed. Although in all clusters in the first
Table II. Correlations between indirect selfdestructiveness and suicide attempt modes

\begin{tabular}{|lccc|}
\hline Suicide attempt modes & Kendall Tau & $Z$ & $p$ \\
\hline Pharmacological drugs & 0.366 & 4.398 & 0.00001 \\
\hline Exsanguination & 0.106 & 1.333 & ns. \\
\hline Hanging & 0.148 & 1.679 & ns. \\
\hline Jumping from a height & 0.092 & 0.829 & ns. \\
\hline Asphyxia & 0.000 & 0.000 & ns. \\
\hline Poisoning & 0.667 & 2.431 & 0.01 \\
\hline $\begin{array}{l}\text { Throwing oneself } \\
\text { under a car }\end{array}$ & 0.542 & 1.975 & 0.04 \\
\hline
\end{tabular}

Table III. CS-DS scores hierarchical cluster analysis

\begin{tabular}{|lccc|}
\hline Cluster & $n$ & Mean & SD \\
\hline 1 (High CS-DS) & 42 & 182.357 & 9.459 \\
\hline 2 (Medium CS-DS) & 48 & 156.062 & 7.141 \\
\hline 3 (Low CS-DS) & 57 & 131.474 & 11.350 \\
\hline
\end{tabular}

position were pharmacological drugs, in the following positions there were different modes for each cluster (of indirect self-destructiveness intensity). Persons with the highest indirect selfdestructiveness intensity prefer exsanguination in the second position; it is likewise for persons with the lowest indirect self-destructiveness intensity, whereas persons in the group with medium indirect self-destructiveness intensity (cluster 2 ) in the second position prefer hanging, one of the most effective modes of committing suicide. All modes of committing suicide appear in cluster 1 , whereas in clusters 2 and 3, poison and throwing oneself under a moving car are absent.

In order to explore differences in choosing particular suicide attempt modes with regard to indirect self-destructiveness intensity, ANOVA (Table V, Figure 1) and post-hoc comparisons were performed (Table VI).

Table IV. Suicide attempt modes within clusters

\begin{tabular}{|c|c|c|c|c|c|c|}
\hline \multirow[t]{2}{*}{ Suicide attempt mode } & \multicolumn{2}{|c|}{ Cluster 1} & \multicolumn{2}{|c|}{ Cluster 2} & \multicolumn{2}{|c|}{ Cluster 3} \\
\hline & $n$ & $\%$ & $n$ & $\%$ & $n$ & $\%$ \\
\hline Pharmacological drugs & 33 & 42.31 & 36 & 41.38 & 30 & 43.48 \\
\hline Exsanguination & 21 & 26.91 & 12 & 13.79 & 27 & 39.12 \\
\hline Hanging & 9 & 11.54 & 24 & 27.59 & 6 & 8.70 \\
\hline Jumping from a height & 6 & 7.69 & 12 & 13.79 & 3 & 4.35 \\
\hline Asphyxia & 3 & 3.85 & 3 & 3.45 & 3 & 4.35 \\
\hline Poisoning & 3 & 3.85 & 0 & 0 & 0 & 0 \\
\hline Throwing oneself under a car & 3 & 3.85 & 0 & 0 & 0 & 0 \\
\hline Total & 78 & 100.00 & 87 & 100.00 & 69 & 100.00 \\
\hline
\end{tabular}


Table V. ANOVA

\begin{tabular}{|lcccccccc|}
\hline Variable & SS effect & $\mathrm{d} f$ effect & MS effect & SS error & $\mathrm{d} f$ error & MS error & $F$ & $p$ \\
\hline Pharmacological drugs & 2.04 & 2 & 1.02 & 30.28 & 144 & 0.210 & 4.861 & 0.009 \\
\hline Exsanguination & 1.80 & 2 & 0.90 & 33.71 & 144 & 0.234 & 3.844 & 0.02 \\
\hline Hanging & 4.21 & 2 & 2.11 & 24.44 & 144 & 0.170 & 12.412 & 0.00001 \\
\hline Jumping from a height & 1.02 & 2 & 0.51 & 16.98 & 144 & 0.118 & 4.303 & 0.01 \\
\hline Asphyxia & 0.01 & 2 & 0.00 & 8.44 & 144 & 0.059 & 0.073 & ns. \\
\hline Poisoning & 0.15 & 2 & 0.08 & 2.79 & 144 & 0.019 & 3.956 & 0.02 \\
\hline $\begin{array}{l}\text { Throwing oneself } \\
\text { under a car }\end{array}$ & 0.15 & 2 & 0.08 & 2.79 & 144 & 0.019 & 3.956 & 0.02 \\
\hline
\end{tabular}

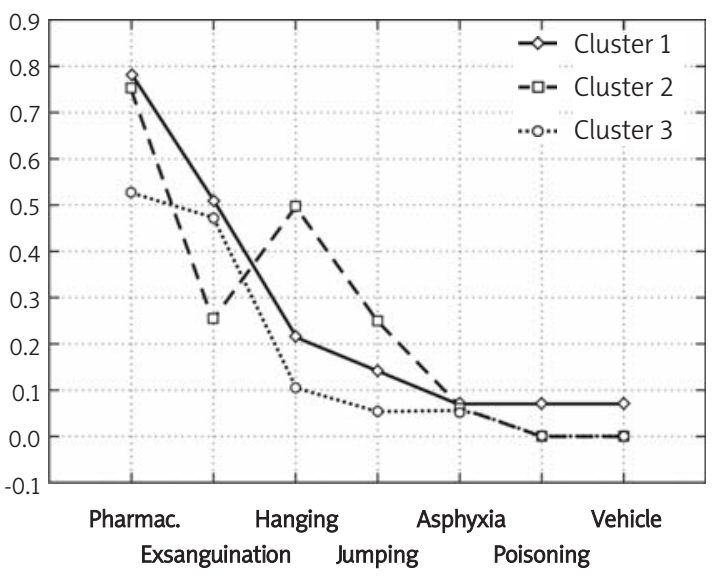

Figure 1. Suicide attempt modes with regard to CS-DS clusters

Table VI. Post-hoc comparisons of cluster scores

\begin{tabular}{|c|c|c|c|}
\hline Pharm. & $\{1\} M=0.786$ & $\{2\} M=0.750$ & $\{3\} M=0.526$ \\
\hline$\{1\}$ & & ns. & 0.02 \\
\hline$\{2\}$ & ns. & & 0.04 \\
\hline$\{3\}$ & 0.02 & 0.04 & \\
\hline Exsang. & $\{1\} M=0.500$ & $\{2\} M=0.250$ & $\{3\} M=0.474$ \\
\hline$\{1\}$ & & 0.04 & ns. \\
\hline$\{2\}$ & 0.04 & & 0.05 \\
\hline$\{3\}$ & ns. & 0.05 & \\
\hline Hang. & $\{1\} M=0.214$ & $\{2\} M=0.500$ & $\{3\} M=0.105$ \\
\hline$\{1\}$ & & 0.004 & ns. \\
\hline$\{2\}$ & 0.004 & & 0.00002 \\
\hline$\{3\}$ & ns. & 0.00002 & \\
\hline Jump. & $\{1\} M=0.143$ & $\{2\} M=0.250$ & $\{3\} M=0.053$ \\
\hline$\{1\}$ & & ns. & ns. \\
\hline$\{2\}$ & ns. & & 0.01 \\
\hline$\{3\}$ & ns. & 0.01 & \\
\hline Poison. & $\{1\} M=0.071$ & $\{2\} M=0.000$ & $\{3\} M=0.000$ \\
\hline$\{1\}$ & & 0.04 & 0.04 \\
\hline$\{2\}$ & 0.04 & & ns. \\
\hline$\{3\}$ & 0.04 & ns. & \\
\hline Car & $\{1\} M=0.071$ & $\{2\} M=0.000$ & $\{3\} M=0.000$ \\
\hline$\{1\}$ & & 0.04 & 0.04 \\
\hline$\{2\}$ & 0.04 & & ns. \\
\hline$\{3\}$ & 0.04 & ns. & \\
\hline
\end{tabular}

Those data show that according to indirect selfdestructiveness intensity, different suicide attempt modes are preferred. Statistically significant differences between clusters were not found only in the case of asphyxia. In order to assess the significance of differences between particular groups, post-hoc comparisons were performed (Table VI).

The greatest difference concerns hanging and jumping from a height, more often undertaken by participants with medium indirect selfdestructiveness intensity (cluster 2); in comparison with individuals from cluster 1 (high intensity) and 3 (low intensity), they most rarely use as a suicide mode exsanguination, which is known to be less effective.

Nevertheless, poisoning and throwing oneself under a moving car occur more often among participants with high indirect self-destructiveness intensity (cluster 1). Moreover, this group is characterized by the greatest variety of suicide attempt modes.

Participants in cluster 3 (low indirect selfdestructiveness intensity) use pharmacological drugs in suicide attempts rarely (in comparison with the other groups).

Concerning the suicide attempts recurrence issue, a correlation was computed which indicates the existence of a relation between indirect selfdestructiveness and suicide attempts recurrence (Kendall Tau: 0.217, $p=0.007$ ). ANOVA reveals that indirect self-destructiveness intensity differentiates risk of consecutive attempts $(F=2.871, p=0.05)$.

Worth noting is that the highest CS-DS score and the highest risk concern cluster 2 (medium indirect self-destructiveness intensity), being significantly higher in comparison with cluster 3 (low CS-DS, $p=0.05)$.

\section{Discussion}

Of interest is the intensity of indirect (chronic) self-destructiveness in the study sample of persons after suicide attempts, containing in the average scores' upper limit; it can be ascribed to the 
effectiveness of therapeutic influences undertaken after the suicide attempt.

It is worth considering that in the study sample, the chosen modes of committing suicide are distinctly different from those found in other studies [10]; perhaps this may be related to the fact that in the mentioned studies suicide completion was studied but not suicide attempts. Furthermore, most of the applied modes (within this work) are characterized by so-called softness (not hardness), low effectiveness and "femininity" rather than "masculinity"; studies state that women "prefer" means of lower effectiveness, such as exsanguination and abuse of hypnotics, while men tend to use firearms and jumping from a height $[10,11]$.

In the present study sample, use of firearms as suicide attempts was not found, perhaps since accessibility to firearms in Poland is very restricted and difficult (culture and legislative specificity?). Keeping firearms at home may be a suicide risk factor, e.g. a suicide attempt over the previous year was common among participants reporting that they currently lived in a home with firearms in USA $[13,14]$.

The existence of associations between indirect self-destructiveness and modes of suicide attempts is beyond any doubt, although an answer to why the mentioned associations concern the most often and the least undertaken modes (pharmacological drugs, poisoning, and throwing oneself under a moving car) may be the subject of further research.

The distribution of modes of suicide attempts within each cluster indicates that high indirect selfdestructiveness intensity is related to the mentioned modes of committing suicide to a large extent. It can be deduced that high indirect selfdestructiveness intensity is associated with the choice of particular suicide attempt modes. In other words, persons with high indirect selfdestructiveness intensity "take the lead", forming the pattern and structure of suicide attempt modes in the whole study population. It can be assumed that persons with the highest indirect selfdestructiveness intensity demonstrate the greatest "creative potential", and the widest spectrum of various suicide attempt modes.

Different modes of suicide attempts are preferred according to the indirect selfdestructiveness intensity.

It can be stated that medium indirect selfdestructiveness intensity is a risk factor for using more efficient suicide modes, of anticipated irreversibility; moreover, in this group, suicide attempts are the most numerous.

Recurrence of suicide attempts according to the indirect self-destructiveness intensity is a distinct issue. As we have seen, there is an association between indirect self-destructiveness and recurrence of suicide attempts, and indirect selfdestructiveness intensity differentiates risk of consecutive attempts. We can see, paradoxically, that persons with medium self-destructiveness intensity are at greater risk of recurrent suicide attempts, rather than persons with high indirect self-destructiveness intensity; paradoxically, the risk of a recurrent (and perhaps effective) suicide attempt is greater in the case of persons with medium indirect self-destructiveness intensity.

The indirect (chronic) self-destructiveness syndrome is often defined as "lingering" or "slow suicide". Persons with that syndrome have a special predisposition to undertake suicide attempts. That may be conditioned by some manifestations of the syndrome, such as helplessness [ $f$ 3] or risky and impulsive behaviours. Concerning helplessness, "learned helplessness", associated with life defeats and self-punishment tendencies, should be mentioned. Consolidated (chronic) helplessness syndrome may cause a threat to life by suicidal tendencies. On the other hand, risky behaviours are connected with the trans-situational pattern of rashness, which assumes the form of indirect selfdestructiveness. A direct condition for suicide attempts may also be impulsive behaviours, defined as a trait of a person and an ego attribute, handicapping in a non-intentional manner behaviour control, in spite of awareness of its threatening results.

In conclusion, the implications of the findings in this work are mainly of preventive and therapeutic nature. In the preventative aspect, the subject of interest should be not only persons with a tendency to become involved in dangerous and aggravated risk situations (active form of indirect selfdestructiveness), but also persons who neglect their own safety or health (passive form of indirect selfdestructiveness). Especially the second form, i.e. the form of negligence, is predominantly ... neglected in preventative and therapeutic work. This is significant, since the risk of recurrent and "effective" suicide attempts is greater not in the case of persons with high indirect selfdestructiveness intensity, but paradoxically in the case of persons with medium self-destructiveness intensity. Neutralisation of risk factors, solving problems and crisis situations, including early diagnosis and therapy, could be important.

Indirect self-destructiveness manifestations as a suicide risk factor may precede the classic prodrome symptoms, e.g. pre-suicidal syndrome according to Ringel [15], and that may activate earlier and facilitate preventative efforts directed at specific individuals.

In the therapeutic activity aspect, it is significant to consider indirect self-destructive tendencies and 
behaviours, especially passive ones (neglect). Confirmation of its importance could be provided by a 10-year follow-up study of adolescents after suicide attempts: it was found that $70.50 \%$ stated they were happy [16]. Moreover, optimistic reframing of negative life events for clients may have treatment implications for the prevention of suicidal activity [17], since it is well known that pessimism is one of the suicide risk factors [18].

A special area of therapeutic efforts may be poor or underdeveloped coping skills, common in indirect self-destructiveness.

Thus neither a suicide attempt itself, nor the suicide attempt mode, erases the possibility of leading a happy life, which is why it is worth offering those persons such help and mobilising them to take the opportunity.

An answer to the question of how many of the suicide attempts were a desperate "cry for help", and how many of them represented actual suicidal intentions, may be the subject of further research.

\section{References}

1. Omigdobun OO, Adejumo OA, Babalola OO. Suicide attempts by hanging in preadolescent children: a case series. West Afr J Med 2008; 27: 259-62.

2. Giegling I, Olgiatib P, Hartmanna AM, et al. Personality and attempted suicide. Analysis of anger, aggression and impulsivity. J Psychiatr Res 2009; in press, available on line 2009.V.28

3. Polewka A, Chrostek-Maj J, Kroch S et al. Poziom poczucia koherencji a ryzyko próby samobójczej. [Sense of coherence and risk of suicide attempt] [Polish]. Przeg Lek 2001; 4: 335-9.

4. Płużek Z. Osobowościowe uwarunkowania pytania o sens życia. [Personality conditions of questions about the meaning of life] [Polish]. In: Popielski K (ed.). Człowiek Wartości - Sens. KUL Lublin 1996; 371-80.

5. Tsirigotis K, Gruszczyński W, Kruszyna M. Autodestruktywność pośrednia u osób po próbach samobójczych. [Indirect self-destructiveness of persons after suicide attempts] [Polish]. Suicydologia 2008; IV: 57-62.

6. Kelley K, Byrne D, Przybyla DPJ, et al. Chronic selfdestructiveness: conceptualization, measurement, and initial validation of the vonstruct. Motivation Emotion 1985; 9: 135-51.

7. Suchańska A. Analisa empirica da fenomeno de autodestrutividade latente. Revista Brasileira de Psychologia 1991; 03: 84-9.

8. Suchańska A. Przejawy i uwarunkowania psychologiczne pośredniej autodestruktywności. [Psychological manifestations and conditions of indirect self-destructiveness] [Polish]. UAM Poznań 1998.

9. StatSoft Polska. Statistica PL. Kraków: StatSoft; 2007.

10. Hołyst B. Samobójstwo. Przypadek czy konieczność. [Suicide - Accident or necessity] [Polish]. PWN, Warszawa 1983.

11. Chambers D. Reach out: Ireland's National Strategy for Action on Suicide Prevention. Suicydologia 2007; III: 12-7.

12. Seligman ME, Walker E, Rosenhan DL. Abnormal psychology. $4^{\text {th }}$ ed. W.W.Norton, New York 2001.
13. Miller M, Barber C, Azrael D, Hemenway D, Molnar BE. Recent psychopathology, suicidal thoughts and suicide attempts in householdswith and without firearms: findings from the National Comorbidity Study Replication. Inj Prev 2009; 15: 183-7.

14. Ohberg A, Lonnqvist J, Sarna S, Vuori E. Violent methods associated with high suicide mortality among the young. J Am Acad Child Adolesc Psychiatry 1996; 35: 144-53.

15. Ringel E. Der Selbstmord. Abschluss einer krankhaften psychischen Entwicklung. Maudrich, Wien-Düsseldorf 1953.

16. Géhin A, Kabuth B, Pichené C, Vidailhet C. Ten year followup study of 65 suicidal adolescents. J Can Acad Child Adolsc Psychiatry 2009; 18: 117-25.

17. Hirsch JK, Wolfdorf K, LaLonde SM, Brunk L, ParkerMorris A. Optimistic explanatory style as a moderator of the association between negative life events and suicide ideation. Crisis 2009; 30: 48-53.

18. Seligman ME. What you can change and what you cannot. Knopf, New York 1994. 